\title{
PRESERVATION OF THE CONSERVATION PROPERTIES OF THE FINITE ELEMENT METHOD UNDER LOCAL MESH REFINEMENT
}

\author{
R.J. LOMAX \\ Electron Physics Laboratory, Department of Electrical and Computer Engineering, \\ The University of Michigan, Ann Arbor, MI 48109, U.S.A.
}

Manuscript received 2 August 1976; Revised manuscript received 6 January 1977

\begin{abstract}
Solutions of partial differential equations obtained by the finite element method can be made to satisfy the same conservation laws as the exact solution. When mesh refinement is carried out, the Galerkin equations must be modified in order to preserve the conservation laws. This modification which arises naturally in the Ritz formulation is described and justified.
\end{abstract}

\section{Introduction}

In general, it is quite difficult to construct finite difference methods for solving partial differential equations which reproduce the conservation laws implied by these equations except in the limit of infinitesimal mesh size. This is particularly true when the boundary conditions are not very simple. However, as has been pointed out by Fix [1-3], properly constructed finite element methods automatically ensurc conservation within the rounding errors of the computation. Although such conservation does not guarantee the accuracy of the results, there is empirical evidence [4] that schemes with this property tend to be more reliable and physically meaningful than alternative methods. In addition, in some situations in which lumped and continuous systems interact, conservation at the interface is highly desirable (e.g. electronic device-circuit interactions [5]).

Another useful feature of the finite element method is the possibility of local mesh refinement. It is important that this be done correctly in order to preserve the conservation properties of the method. This paper discusses how the conservation properties may be retained when local mesh refinement is carried out.

\section{Conservation properties}

Consider the solution of the partial differential equation

$$
L u-f(\boldsymbol{x}, t)=0,
$$

where $L$ is a partial differential operator, $u(x, t)$ is the unknown, and $f(x, t)$ is a given forcing function. The solution is defined in a two-dimensional region $\Omega$ bounded by a curve $\Gamma$. For simplicity it will be assumed that the boundary conditions on $u$ are Dirichlet on some parts of $\Gamma$ and 
Neumann elsewhere. Conservation laws can be derived by taking the inner product of eq. (1) and some function $v(\boldsymbol{x}, t)$ over the region $\Omega$ :

$$
(L u-f, v)=0 .
$$

In general, eq. (2) can be transformed by integration by parts to

$$
a(u, v)=0 \text {, }
$$

where $a(u, v)$ is a function linear in $v$ which will involve certain line integrals along $\Gamma$ arising from the partial integration. These correspond physically to the fluxes of certain quantities through the boundary.

If a set of basis functions $\left\{\phi_{j}(\boldsymbol{x})\right\}$ for the finite element approximation $u^{h}$ is introduced, the Galerkin finite element equations are given by the corresponding functional evaluated for the basis functions

$$
a^{h}\left(u^{h}, \phi_{j}\right)=0, \quad j=1,2, \ldots N_{p},
$$

where $N_{p}$ is the number of nodes excluding those for which Dirichlet boundary conditions are specified; inner products are taken over $\Omega^{h}$, the element approximation to $\Omega$; and line integrals are along $\Gamma^{h}$, the element approximation to $\Gamma$. If $N_{p+1}, N_{p+2}, \ldots N$ represent the remaining nodes at which Dirichlet conditions are imposed, eq. (4) will be extended to these nodes also. This serves to define the fluxes arising from the line integrals along $\Gamma$ in an unambiguous way. It then follows from the extended eq. (4) that

$$
a^{h}\left(u^{h}, v^{h}\right)=0,
$$

for any $v^{h}$ belonging to the space spanned by the basis functions. In particular, if $v$ used in the conservation law eq. (3) lies in this space (i.e. $v \equiv v^{h}$ ), and if $\Omega^{h}, \Gamma^{h}$ are identical to $\Omega, \Gamma$, then the same conservation law holds exactly for $u^{h}$ as held for $u$ (compare eqs. (3) and (5)). The assumption that $\Omega^{h}, \Gamma^{h}$ are identical to $\Omega, \Gamma$ requires that the region can be divided up exactly into finite elements. This will be true if $\Omega$ is a polygon or under certain conditions when isoparametric elements are used.

For more general boundary conditions, a hybrid formulation can be used (see Fix [1]).

\section{Local mesh refinement}

The finite element method lends itself to relatively straightforward implementation of local mesh refinement $[6,7]$. It is important, however, not to destroy the conservation properties when this is done. Fig. 1 shows two stages of mesh refinement in the vicinity of point $P$ in a square mesh (Notc that the following discussion is general and applics to any clement shapc.) At the interface of the original or coarse mesh and the refined mesh, there will be nodes which belong to the refined elements but not the coarse ones. These are labeled D1 and D2 in fig. 1 and will be called dummy nodes. Remaining nodes will be called regular nodes. The values of the variables at dummy 


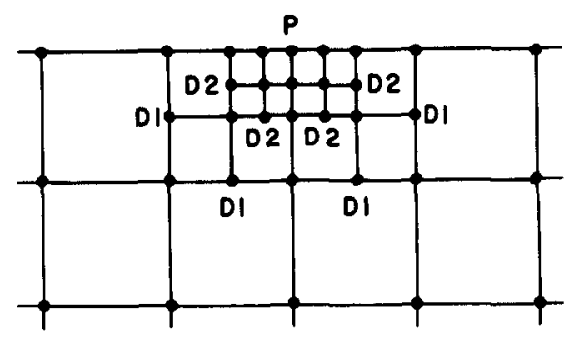

Fig. 1. Square mesh with two stages of refinement showing dummy nodes D1 and D2.

nodes are determined directly by using the finite element approximation obtained from the less refined elements, and they do not depend explicitly on the particular equations being solved. The values of the variables at all nodes can be grouped together into a vector $\boldsymbol{u}$, and the subset of regular nodes can be grouped together into a vector $\boldsymbol{u}_{r}$. At each level of refinement the new dummy nodal values of $\boldsymbol{u}$ can be expressed as linear combinations of all the previously defined values, regular and dummy. Thus by successive elimination all nodal values $\boldsymbol{u}$ can be determined as a linear combination of the $\boldsymbol{u}_{r}$ by an expression of the form

$$
\boldsymbol{u}=\boldsymbol{Q} u_{r}
$$

\section{Effect of refinement on conservation}

From eq. (6) it follows that $u^{h}(\boldsymbol{x})$ can be expressed as

$$
u^{h}(\boldsymbol{x})=\boldsymbol{\phi}^{\mathrm{t}} \boldsymbol{u}=\boldsymbol{\phi}^{\mathrm{t}} \boldsymbol{Q} \boldsymbol{u}_{r},
$$

where $\phi^{t}$ is the transpose of the vector with components $\phi_{i}$. The set of Galerkin equations (4) becomes

$$
a^{h}\left(\phi^{\mathrm{t}} Q u_{r}, \phi\right)=0
$$

However, this is now overdetermined, for $\boldsymbol{u}_{r}$ contains fewer components than $\boldsymbol{u}$ and $\boldsymbol{\phi}$. A determinate set is obtained by premultiplication of eq. (8) by $Q^{t}$ :

$$
a^{h}\left(\phi^{\mathfrak{t}} Q u_{r}, Q^{\mathbf{t}} \phi\right)=0 .
$$

(This approach arises naturally in the Ritz formulation of the finite element method by viewing the problem as a constrained minimization and using undetermined Lagrangian multipliers or direct substitution to take account of constraints $[7,8]$.) Equation (9) is determinate because the column dimension of $\boldsymbol{Q}^{\mathrm{t}}$ is the same as the dimension of $\boldsymbol{u}_{\boldsymbol{r}}$. An apparently simpler procedure would be to use eq. (8) only for the shape functions $\phi_{j}$ corresponding to regular nodes. However, this can be shown to be nonconservative. It will now be demonstrated that eq. (9) preserves the conservation laws.

The conservation law corresponding to eq. (5) may be derived by substituting 


$$
v(\boldsymbol{x})=v^{h}(\boldsymbol{x}) \mathbf{\phi}^{\mathbf{t}} \boldsymbol{v}
$$

for appropriate constant $v$, i.e.

$$
a^{h}\left(\phi^{\mathrm{t}} u, \boldsymbol{\phi}^{\mathrm{t}} v\right)=0
$$

This is derivable from the modified finite element equations, eq. (9), if there is a linear combination of the set eq. (9) such that it gives the same result as eq. (11). Let the required linear combination of eq. (9) be

$$
a^{h}\left(\phi^{\mathrm{t}} Q u_{r}, \phi^{\mathrm{t}} Q p\right)=0,
$$

for appropriate constant $\boldsymbol{p}$, then, because of eq. (7), eqs. (11) and (12) are equivalent if

$$
\phi^{\mathrm{t}} v=\phi^{\mathrm{t}} Q p
$$

Since $\boldsymbol{\phi}$ is composed of a linearly independent set of functions, this requires

$$
v=Q p
$$

In general, eq. (14) is an overdetermined set of equations for $\boldsymbol{p}$; however, it has a solution when $v(\boldsymbol{x})$ is restricted to functions which can be expressed exactly in the subspace whose basis is the set of functions $Q^{t} \phi$ obtained from linear combinations of the original complete set of shape functions $\phi$, i.e. if $v=\phi^{t} Q \hat{v}$, then $v=Q \hat{v}$ and eq. (14) has the solution $\boldsymbol{p}=\hat{\boldsymbol{v}}$.

Two special cases are of particular importance in deriving conservation laws:

(a) $v(\boldsymbol{x}) \equiv 1$. This usually gives the most commonly recognized conservation laws (e.g. conservation of mass, charge etc.).

(b) $v(\boldsymbol{x})=u^{h}(\boldsymbol{x})$. In this case $\boldsymbol{v}=\boldsymbol{u}$ and $\boldsymbol{p}=\boldsymbol{u}_{r}$.

Case (a) implies that the conservation law derived from the original partial differential equation is still valid provided eq. (9) is used to define the finite element equations. Case (b) is of importance in some conservation laws involving quadratic expressions [1].

\section{Conclusions}

Under boundary conditions which are piecewise Dirichlet or Neumann everywhere the property of the finite element method of preserving an important class of conservation laws is maintained under mesh refinement provided the equations are set up in the manner described.

From these results it is clear that the introduction of special elements as proposed for example by Hachtel et al. [8] is not necessary to preserve global conservation even when $C^{0}$ elements are used. Provided that fluxes are again defined in accordance with the above scheme, conservation is also obtained over an individual element. This avoids the introduction $[9,10]$ of a region of different shape from the element over which conservation is valid. 


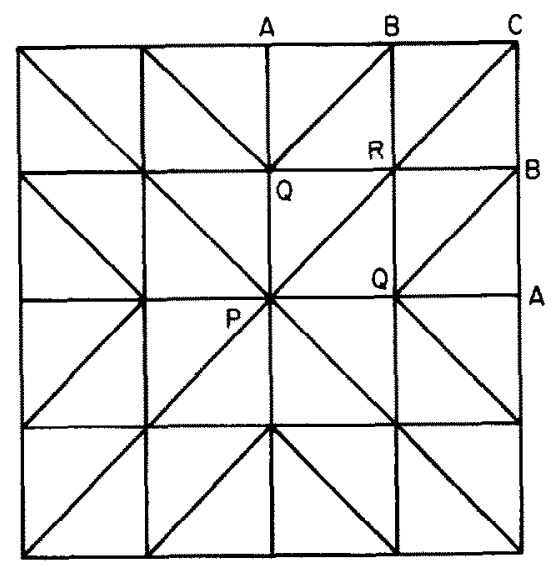

(a)

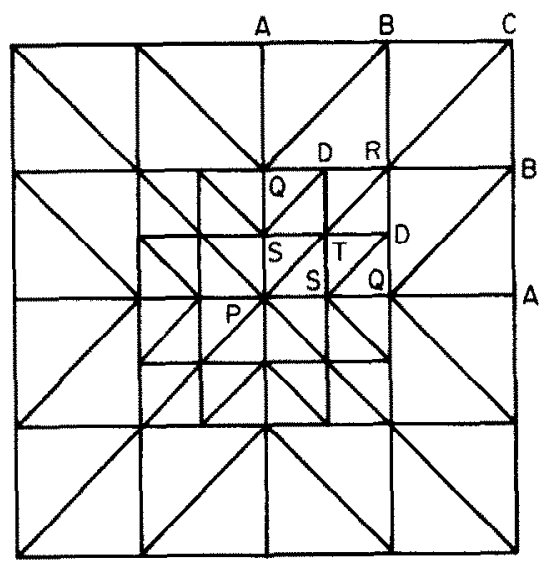

(b)

Fig. 2. (a) Uniform mesh for solution of Poisson's equation. (b) Refined mesh with dummy nodes $D$.

\section{Acknowledgment}

This work was supported by the National Science Foundation under Grant No. ENG 75-12833.

\section{Appendix}

As a simple example of the effect of mesh refinement, consider the solution of $\nabla^{2} u=-1$ on the unit square with $u=0$ on the edges of the square, i.e. $u_{\mathrm{A}}=u_{\mathrm{B}}=u_{\mathrm{C}}=0$ in figs. $2 \mathrm{a}$ and $2 \mathrm{~b}$. In fig. $2 b, D$ represents the dummy nodes. (Only the first quadrant is lettered - the other points may be determined by symmetry.) For linear shape functions the finite element equations can be solved exactly to yield the results shown in table 1 . Also given in this table are the fluxes of $\nabla u$, namely $F_{A}, F_{B}$ and $F_{C}$, which are associated with nodes lettered $A, B$ and $C$. The net flux is therefore

$$
4 F_{\mathrm{A}}+8 F_{\mathrm{B}}+4 F_{\mathrm{C}}=-1
$$

This is in agreement with the exact solution

$$
\int_{\Gamma} \nabla u \cdot n \mathrm{~d} S=\int_{\Omega} \nabla^{2} u \mathrm{~d} A=\int_{\Omega}(-1) \mathrm{d} A=-1 .
$$

Table 1. Finite element solution of Poisson's equation

\begin{tabular}{llllllllll} 
Case & $u_{\mathrm{D}}$ & $u_{\mathrm{P}}$ & $u_{\mathrm{O}}$ & $u_{\mathrm{R}}$ & $u_{\mathrm{S}}$ & $u_{\mathrm{T}}$ & $F_{\mathrm{A}}$ & $F_{\mathrm{B}}$ & $F_{\mathrm{C}}$ \\
\hline $\begin{array}{l}\text { Fig. 2a } \\
\text { (without dummy nodes) }\end{array}$ & & $\frac{330}{4224}$ & $\frac{242}{4224}$ & $\frac{187}{4224}$ & - & - & $-\frac{330}{4224}$ & $-\frac{319}{4224}$ & $-\frac{88}{4224}$ \\
$\begin{array}{l}\text { Fig. 2b } \\
\text { (with dummy nodes) }\end{array}$ & $\frac{215}{4224}$ & $\frac{312}{4224}$ & $\frac{244}{4224}$ & $\frac{186}{4224}$ & $\frac{290}{4224}$ & $\frac{269}{4224}$ & $-\frac{332}{4224}$ & $-\frac{318}{4224}$ & $-\frac{88}{4224}$ \\
\hline
\end{tabular}




\section{References}

[1] G.J. Fix, Hybrid finite element methods, SIAM Rev. 18 (1976) 460-484.

[2] C.J. Fix, Finite element models for ocean circulation problems, SIAM J. Appl. Math. 29 (1975) 371 - 387.

[3] G.J. Fix, Finite elements and fluid dynamics, in: J.T. Oden (ed.) Computer methods in nonlinear mechanics (Springer-Verlag, New York, 1975).

[4] P.J. Roache, Computational fluid dynamics (Hermosa Publishers, Albuquerque, NM) $28-33$.

[5] J.J. Barnes and R.J. Lomax, Finite element methods in semiconductor device simulation. To appear in IEEE Trans. Electron Devices ED-24 (1977).

[6] I.J. Somervaille, A technique for mesh grading applied to conforming plate bending finite elements, Int. J. Numer. Meths. Eng. 7 (1973) 310-312.

[7] G.F. Carey, A mesh-refinement scheme for finite element computations, Comp. Meths. Appl. Mech. Eng. 7 (1976) $93-105$.

[8] G.D. Hachtel, M. Mack and R.R. O'brien, Semiconductor device analysis via finite elements, Conference Record, Eighth Asilomar Conference on Circuits, Systems and Computers, Pacific Grove, Monterey, CA (1974) $332-338$.

[9] E.M. Buturla and P.E. Cottrell, Steady state analysis of ficld effect transistors via the finite clement method, IEEE International Electron Devices Meeting Technical Digest, Washington DC (1975) $51-54$.

[10] G.D. Hachtel, M.H. Mack, R.R. O’Brien and H.F. Quinn, Two-dimensional finite element modeling of NPN devices, IEEE International Electron Devices Meeting Technical Digest, Washington DC (1976) $166-169$ 